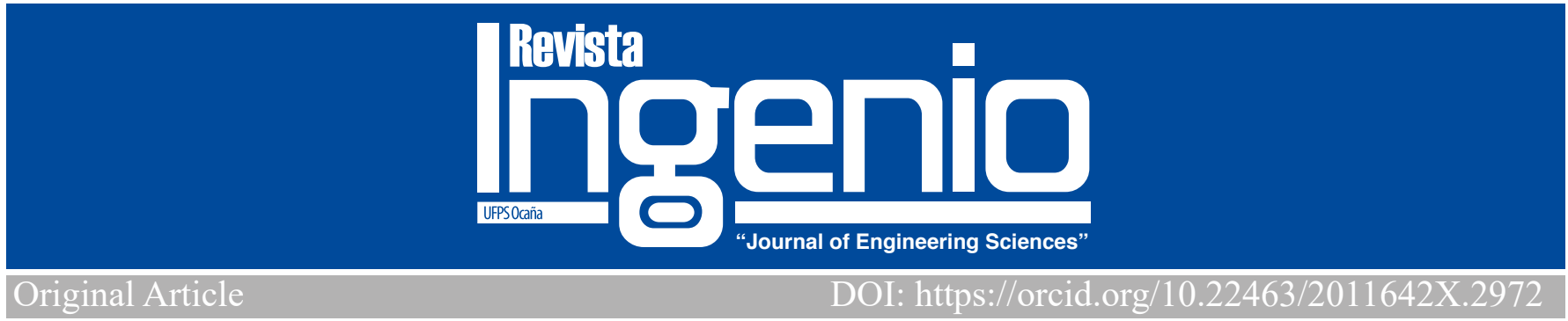

\title{
Estudio numérico del proceso de cocción de ladrillos al interior de un horno tipo Hoffman
}

\author{
Numerical study of burning brick inside Hoffman kiln \\ MSc. Hernando Alexander Yepes Tumay \\ ${ }^{I}$ Grupo de Investigación en Nuevas Tecnologias, Sostenibilidad e Innovación (GINSTI), Departamento de Ingenieria Mecánica, Universidad Francisco de Paula Santander - Ocaña, Colombia, https://orcid.
} org/0000-0001-9399-5673, Email: hayepest@gmail.com

Cómo citar: H. A. Yepes, "Estudio numérico del proceso de cocción de ladrillos al interior de un horno tipo Hoffman”,Revista Ingenio, vol.15 (1), pp. $8-15,2018$.

Fecha de recibido:09 de julio de 2018 Fecha aprobación: 26 de octubre de 2018

\section{RESUMEN}

Palabras claves:

El aumento en la demanda energética y las emisiones contaminantes, así como la dependencia de los combustibles fósiles a nivel mundial, genera la necesidad de buscar estrategias enfocadas en una mayor eficiencia y mejoramiento en los procesos de transformación energética. En este estudio se evaluó numéricamente el proceso de cocción de ladrillos al interior de un horno tipo

CFD, Flujo de fluido, Horno Hoffman, Cocción Hoffman, utilizando un enfoque de dinámica de fluidos computacionales (CFD), analizando los campos de temperatura y los patrones fluidodinámicos al interior del proceso. Los resultados obtenidos además de analizar en detalle cómo se dan los fenómenos térmicos al interior del horno evidenciaron varios campos de estudio en los que se debe profundizar para lograr una mejora energética en la cocción de ladrillos y por tanto mayor eficiencia.

\section{ABSTRACT}

Key words:

CFD, Fluid flow, Hoffman

kiln, brick firing.

The growth of energy requirements, pollutant emissions and fossil fuel dependency at the global level require strategies to achieve higher efficiency and improvements in the energy transformation process. The brick burning process at Hoffman kiln was evaluated through CFD simulation, flow and thermal profiles were analyzed. Additionally, to the thermal phenomena inside the furnace, the results allowed to identify some topics that require a more profound study to improve the brick burning process from an energetic point of view and, therefore, higher efficiency.

\section{Introducción}

El crecimiento poblacional, los nuevos estándares de vida, el aumento de la economía y los avances tecnológicos han generado un gran incremento energético a nivel mundial. De acuerdo a la Agencia Internacional de la Energía (IEA por sus siglas en inglés) la demanda mundial aumentará en un 14.6\% para el año 2020 y un $48 \%$ para el 2040 en comparación a la energía requerida por la población en 2012, pasando de 549 cuatrillones de BTU a 815 en dicho periodo de tiempo [1]. América Latina no es ajena a esta tendencia e incluso en los últimos 40 años ha aumentado su consumo energético en cerca de 2.5 veces, con un crecimiento promedio anual de $8 \%$. En el caso colombiano de acuerdo con el Banco Interamericano de Desarrollo para el año 2040 el incremento en el consumo energético será de $110 \%$ en comparación al año 2013 con un aumento anual promedio de $2.8 \%$. [2].
Una de las estrategias para reducir el consumo los combustibles fósiles por sus efectos negativos y de igual forma contribuir al cumplimiento de la demanda energética, es promover la optimización de procesos que permitan un mejor aprovechamiento de los recursos energéticos. En el caso Colombiano y de América Latina este aspecto es de vital consideración dado que por ser en su mayoría países en vía de desarrollo, la disponibilidad y facilidad de implementación de tecnologías eficientes resulta complicada en comparación a otros países [3]. En particular el sector industrial colombiano y específicamente en procesos térmicos se presenta un rezago tecnológico que deriva en un uso ineficiente de energía lo que incrementa los consumos [4-5-22]. Dentro de estos uno de los procesos de mayor requerimiento energético es la cocción de cerámicos [6].

El uso de CFD para la optimización de hornos y sistemas 
energéticos es cada vez mayor debido a las ventajas de esta técnica, donde se pueden realizar análisis detallados de variables que son experimentalmente muy difíciles de cuantificar. Diversos estudios han usado este enfoque para establecer mejoras dentro de diferentes procesos térmicos. Con el objetivo de obtener un modelo para sustentar propuestas de mejoramiento viables para la industria cerámica Possamai et al. [7] llevaron a cabo el modelado de un horno para la producción de vidrio pulverizado, evaluando tres grados diferentes de aislamiento. El modelo permitió identificar puntos calientes en las paredes e identificar un movimiento ligero de la llama hacia uno de los costados del horno debido a la ubicación de la salida de gases.

Ubaque et al [8] adelantaron un estudio numérico de un horno tipo Hoffman de ladrillos para encontrar los puntos óptimos de temperatura con el objetivo de incinerar residuos sólidos. Los resultados permitieron identificar las zonas de mayor turbulencia para ubicar los residuos, las cuales dependen de la ubicación de los ladrillos. Se pudo también comprobar de manera preliminar que la ubicación de los desechos no afecta el proceso de cocción. En la misma línea de optimizar el proceso de combustión Tu et al [9] llevaron a cabo un estudio numérico sobre el efecto de la forma de la cámara de combustión, donde encontraron que al aumentar el ángulo de inclinación del techo y suelo de la cámara se genera una mayor recirculación y por tanto se obtiene una zona de reacción mucho más amplia con menores temperaturas y bajas emisiones de NOx Liu et al [10] utilizaron la herramienta CFD para estudiar las características de calentamiento y uniformidad de losas de acero en un horno de recalentamiento. Los resultados permitieron evidenciar que durante el calentamiento de las losas se generan altos gradientes de temperatura debido a la inferioridad del flujo de aire.

Bajo estas consideraciones se plantea el presente estudio, en el cual se espera evaluar numéricamente unhorno industrial para la fabricación de ladrillos tipo Hoffman, mediante la modelación del proceso de cocción utilizando CFD, analizando específicamente los patrones fluidodinámicos y campos de temperatura al interior del horno.

\section{Metodología}

\subsection{Horno Hoffman}

El presente estudio se llevó a cabo tomando como dominio el horno de la empresa "Ladrillera Ocaña". El sistema está constituido por dos secciones rectangulares de $44.1 \mathrm{~m}$ de largo, cada una de ellas está divida en 12 cámaras donde son ubicados los ladrillos, cada cámara cuenta una puerta de principal para la carga y descarga de estos, además de una compuerta para el ingreso de aire y la extracción de gases; mayores detalles pueden encontrarse en [11]. El esquema del funcionamiento de este tipo de hornos se muestra en la Figura 1. Cada cámara tiene la capacidad para almacenar 5 apiles, los cuales consisten en un arreglo organizado de aproximadamente 600 ladrillos en dos paneles.

Transversalmente el arreglo permite el flujo de gases por la parte superior y a través de 6 canales en la parte inferior. Los denominados apiles se posicionan con una distancia de separación de $60 \mathrm{~cm}$.

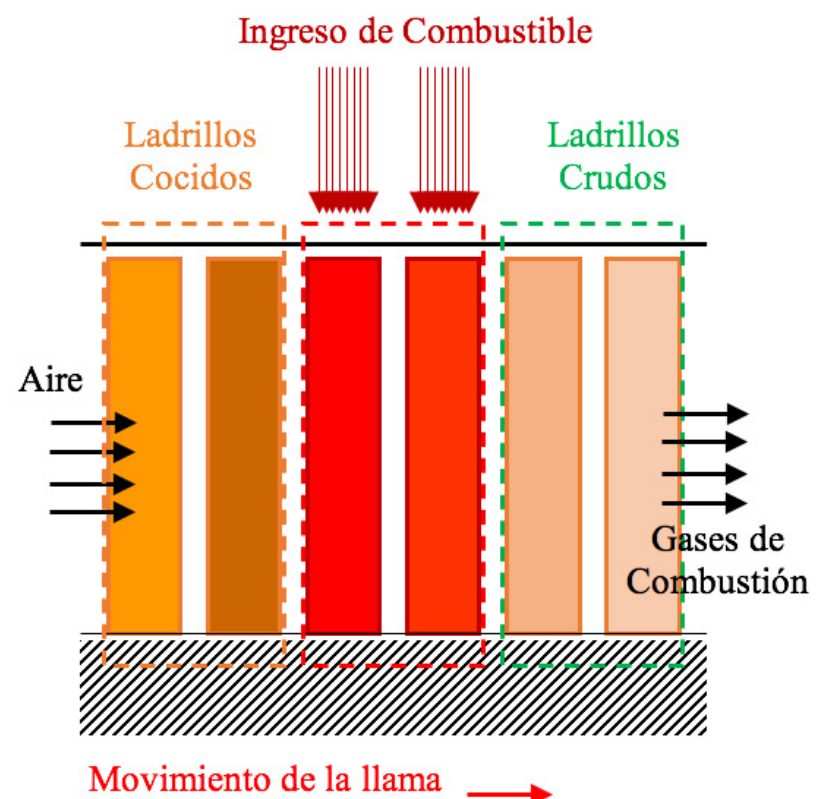

Figura 1. Esquema de funcionamiento horno Hoffman.

\subsection{Dominio computacional}

La simulación se realizó usando el programa Ansys-Fluent 19.0. Considerando el arreglo de los ladrillos, la cantidad de apiles y que las condiciones en cada cámara se pueden considerar informes, el estudio de una sola de estas, es una representación acorde de los procesos que se dan en la totalidad el horno. Bajo esta premisa el dominio de simulación fue acotado a una longitud de $5.4 \mathrm{~m}$ lo cual abarca una cámara completa $(4.9 \mathrm{~m})$ y una zona de consideración al comienzo y al final para el desarrollo del flujo, junto con los ladrillos ubicados en su interior.

Con el objetivo de abordar tanto el efecto de calentamiento al interior de los ladrillos como la interacción de los elementos presentes dentro de la cámara, en las simulaciones desarrolladas se consideró cada uno de los apiles completos como toda una zona maciza, siendo un total de 4 apiles en 
la longitud de dominio determinada. Los detalles presentes en los ladrillos fueron omitidos, con el fin de evitar un excesivo número de celdas y por tanto un incremento en el costo computacional. Si bien en algunos estudios como el desarrollado por Ubaque y colaboradores [8] implementan estos detalles, utilizan simetrías junto con celdas tetraédricas, las cuales incrementan la incertidumbre numérica, mientras en el presente estudio se usaron únicamente celdas hexaédricas.

Las puertas de carga y de ingreso de aire, se encuentran cerradas al momento del calentamiento y la cocción, etapas que son el enfoque del presente estudio, por lo cual son omitidas, es decir que la pared lateral donde estarían ubicadas se considera completamente plana. Si bien ambas compuertas al tener un espesor menor al de las paredes del horno pueden incidir en la transferencia de calor por conducción que tiene lugar a través de estas, su área de interacción es mucho menor al total de las fronteras por lo cual la incidencia puede considerarse muy pequeña.

\subsection{Mallado del dominio}

Para la simulación se construyó un mallado con 2.062.945 de celdas hexaédricas, el cual cuenta con un Equiangle Skewness(Eqsk) máximo de 0.67. La distribución entre el número de celdas junto con el rango de valores de este parámetro y su apreciación cualitativa de calidad [12] se muestran en la Tabla 1.

Tabla 1: Distribución de celdas y calidad del mallado

\begin{tabular}{lll}
\hline Celdas & $E_{q s k}$ & Calidad \\
\hline $1.796 .983(87.11 \%)$ & $0.0-0.25$ & Excelente \\
$248.595(12.05 \%)$ & $0.25-0.50$ & Buena \\
$17.367(0.84 \%)$ & $0.50-0.75$ & Aceptable
\end{tabular}

De esta manera el mallado construido cuenta con un $99.16 \%$ de la totalidad de sus celdas con un nivel de calidad excelente o buena, donde incluso la mayoría está catalogada con el más alto estándar, lo cual permite establecer una adecuada discretización espacial que permite evitar problemas de solución a nivel numérico durante la simulación. Adicionalmente el incremento en el tamaño de celda usado no supera el $20 \%$. El dominio computacional con su mallado se muestra en la Figura 2.

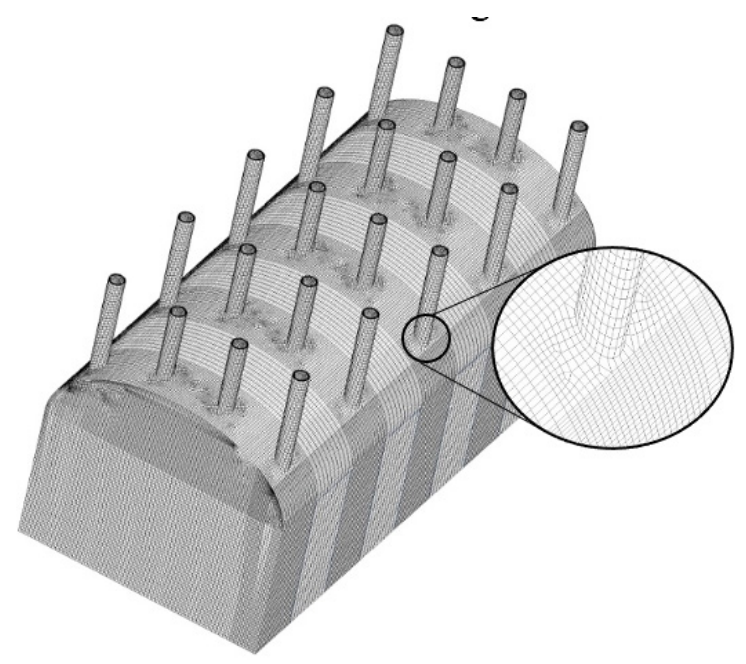

Figura 2. Mallado hexaédrico.

Por otra parte, en las zonas cercanas a las paredes se utilizó un mallado fino para lograr captar le efectos de capa limite y los gradientes que se forman cerca de las interfaz sólido - fluido. En la Figura 3 se muestran los refinamientos en parte de las zonas mencionadas.

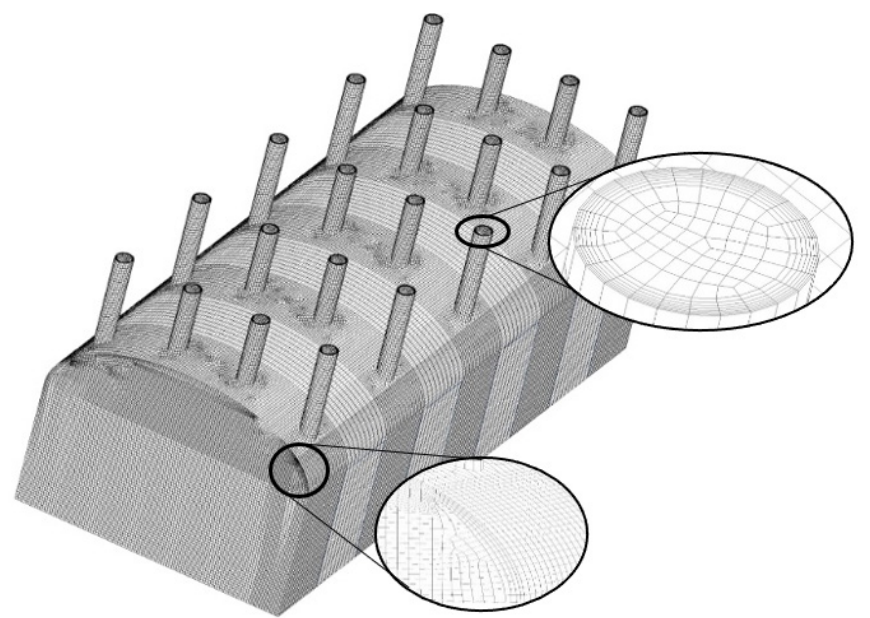

Figura 3. Refinamiento para las capas límite en las fronteras.

De igual forma los ladrillos al interior del horno también fueron incluidos dentro del dominio y por tanto fueron mallados. En la Figura 4 se muestra los bloques constituidos por las celdas hexaédricas.

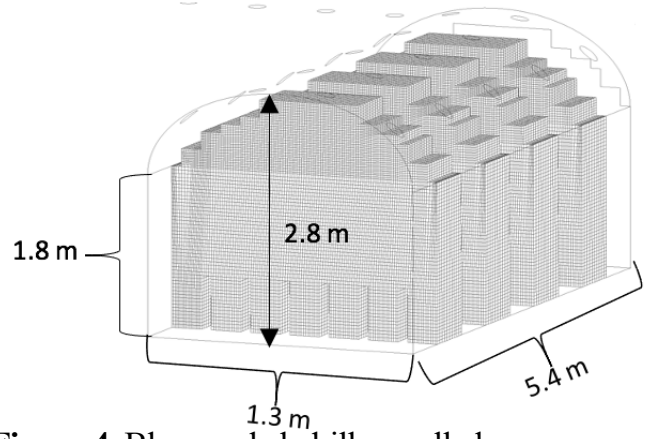

Figura 4. Bloques de ladrillos mallados. 


\subsection{Mallado del dominio}

Las dimensiones generales del dominio computacional se muestran en la Figura 3, los detalles pueden encontrarse en [11]. Para las simulaciones desarrolladas se utilizaron 3 tipos de condiciones de frontera: flujo másico de entrada, presión de salida y pared. En las fronteras de esta última tipología se especificaron diferentes condiciones térmicas de acuerdo a lo reportado en los estudios previos [11-13]. En la Figura 5 se muestra de forma esquemática las diferentes condiciones de frontera usadas durante la simulación con un esquema de identificación por color. El color azul denota las entradas al dominio, el color rojo señala las salidas de este y el color blanco está asociado con las fronteras de tipo pared. Tal como se puede apreciar existe una sola entrada y salida del dominio considerado, sin embargo, existen diferentes tipos de pared dada su ubicación y condiciones térmicas.

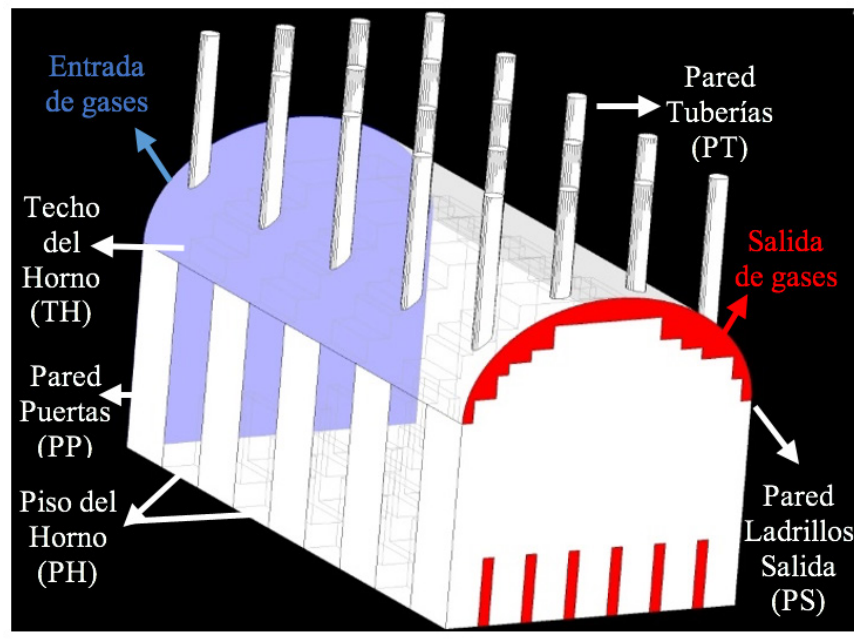

Figura 5. Condiciones de frontera en la simulación.

La entrada de gases corresponde a los productos de combustión que ingresan al dominio proveniente de las cámaras precedentes. Para determinar el valor del flujo másico correspondiente y la composición de estos se utilizó los datos reportados por Guerrero [11]. Considerando una potencia de operación de $4310.2 \mathrm{~kW}$ y un factor de aireación de 1.76, el valor del flujo másico de aire fue tomado para la condición de frontera "Entrada de gases", si bien es cierto que el flujo real puede ser ligeramente mayor debido a los volátiles liberados durante la combustión del carbón y su consumo mismo, esto representa una fracción muy pequeña en comparación al flujo de aire. De manera explícita el proceso de combustión del carbón tampoco fue considerado en las simulaciones realizadas ya que determinar la cantidad exacta de volátiles, así como la tasa de liberación de forma precisa, el análisis último para determinar la composición química y tamaño de partícula supone un alcance que va más allá de lo abordado en la presente investigación. El efecto del combustible y su descarga sobre los apiles considerados en la cámara fue tenida en cuenta mediante la variación de la temperatura. Esta aproximación es comúnmente usada en procesos de simulación debido al requerimiento computacional que implica la consideración del proceso de combustión.

La composición de los gases de combustión fue determinada usando los valores promedio de las mediciones realizadas por Guerrero [11] en la chimenea del horno, estableciendo una composición de 7.12\% CO2, 5.32\% H2O, $6.46 \% \mathrm{O} 2,4.9 \%$ CO y $76.20 \% \mathrm{~N} 2$ ( $\%$ en volumen).

Para la superficie denotada "Salida de gases" se utilizó una condición de presión de salida que se fijó en un valor negativo de $10.000 \mathrm{~Pa}$, emulando la presión de succión que se genera dentro del horno producto del extractor. Tanto en las entradas como salidas se usó el diámetro hidráulico y la intensidad de turbulencia (IT) como condiciones de frontera para el flujo. Los valores calculados de IT oscilaron alrededor de $5 \%$ por lo que se fijó este valor para todas las fronteras.

En la Tabla 2 se establecen las disposiciones térmicas fijadas en las fronteras tipo pared. En la columna sigla, las dos primeras letras hacen referencia a la pared de acuerdo con lo expuesto en la Figura 5 y PO indica la pared opuesta a la Pared Puertas. La tercera hace referencia al tipo de condición, donde $\mathrm{C}$ indica convección, A si se considera adiabática y $\mathrm{T}$ si es de temperatura fija.

Tabla 2: Disposiciones térmicas en las paredes.

\begin{tabular}{ccccc}
\hline Sigla & $\begin{array}{c}\text { Espesor } \\
(\mathrm{m})\end{array}$ & $\begin{array}{c}\mathrm{Ts} \\
(\mathrm{K})\end{array}$ & $\begin{array}{c}\mathrm{h} \\
\left(\mathrm{W} / \mathrm{m}^{2}-\mathrm{K}\right)\end{array}$ & $\mathrm{T}_{\infty}(\mathrm{K})$ \\
\hline PP-C & 0.9 & - & 10 & 300 \\
PH-A & 0.15 & - & - & - \\
PT-A & - & - & - & - \\
PS-C & 0.6 & - & 5 & 300 \\
PO-C & 1.2 & - & 5 & 300 \\
TH-T & 1.2 & 307.6 & - & - \\
\hline
\end{tabular}

Los valores de $\mathrm{h}$ fueron fijados a partir de datos reportados en la literatura, congruentes para una convección natural [16-17]. En PO se asumió un valor menor, dado que ésta tiene como espacio libre para el fluido las otras cámaras del horno que si bien no están en funcionamiento el flujo de aire y por tanto la velocidad es mucho menor. El piso del horno fue considerado adiabático de acuerdo a lo reportado en diferentes estudios [18-19], mientras que las paredes de los tubos se considera esta misma condición dado que están inmersos dentro del ladrillo cerámico lo que permite un 
aislamiento bastante alto, permitiendo que la aproximación considerada sea apropiada.

\subsection{Modelos complementarios}

El tensor de Reynolds fue calculado usando el modelo $\kappa-\varepsilon$ junto con las funciones de pared estándar. La selección de este modelo se hizo basado en los resultados adecuados obtenidos en diversas investigaciones ,[18]. Para la transferencia de calor por radiación el modelo P1 fue seleccionado debido a su buen desempeño en estudios anteriores [19] y simplicidad computacional. Las propiedades de radiación como la absortividad fueron determinadas usando el modelo de suma ponderada de gases grises (wsggm) el cual es adecuado cuando se tienen mezclas como es el caso de los productos de combustión.

\subsection{Procedimiento de simulación}

La simulación se llevó a cabo en estado transitorio para evaluar el proceso de calentamiento. El número de Courant convectivo en cada celda fue menor a 40 en todo el dominio. Para tener en cuenta las etapas descritas en la Figura 1, esta fue dividida en tres fases. La primera abarca los efectos iniciales hasta el acercamiento medio de la llama y por tanto un incremento moderado en la temperatura de los gases de combustión. La segunda tiene encuentra el momento donde la llama se encuentra en las inmediaciones de la cámara lo que produce un incremento de temperatura de los gases y finalmente la etapa de cocción donde la zona de reacción se encuentra en la cámara y por tanto la temperatura es máxima.

En la primera fase se hizo un aumento paulatino de los gases de combustión desde $343 \mathrm{~K}$ hasta $473 \mathrm{~K}$ en un periodo de tiempo total de 1.2 horas. Para la segunda fase la temperatura de los gases fue incrementada a un $60 \%$ de la temperatura de llama adiabática del combustible; el periodo de tiempo para esta etapa fue de 5 horas. Finalmente, en la tercera fase se considera un incremento de $200 \mathrm{~K}$ en la temperatura de los gases debido a la presencia de la zona de reacción en la cámara, la cual se realizó durante un tiempo de 8.9 horas.

En cada paso de tiempo de la simulación se realizaron 20 iteraciones con el fin de garantizar la convergencia, de igual forma se tomó como criterio de convergencia que los residuales para la energía y la radiación estuviesen en el orden de 10-6 o menor, en el caso de las velocidades se tomó de $10-4$, mientras que para la continuidad el valor fue de 10-5 [21].

\subsection{Independencia de mallado}

Las simulaciones de comparación se realizaron usando 3 mallados diferentes. En la Tabla 3 se muestra el número de celdas y la zona específica de refinamiento de cada uno de los mallados usados. La selección de las zonas de refinamiento se hizo tomando como criterio las regiones de formación de capas límite y altos gradientes tanto de velocidad como de temperatura que pudiese afectar los resultados de la simulación.

Tabla 3: Número de celdas de los mallados usados.

\begin{tabular}{lll}
\hline Mallado & $\begin{array}{c}\text { Número de } \\
\text { celdas }\end{array}$ & Zona de refinamiento \\
\hline Grueso & 2.062 .945 & - \\
Intermedio & 2.205 .542 & $\begin{array}{l}\text { Celdas aledañas a los } \\
\text { bloques de ladrillos. }\end{array}$ \\
Fino & 2.386 .226 & $\begin{array}{l}\text { Celdas en la zona } \\
\text { superior del horno } \\
\text { aledañas al techo. }\end{array}$
\end{tabular}

Para establecer la independencia del mallado se compararon las temperaturas y velocidades en diferentes puntos del horno tanto en la zona del fluido como en la región sólida. De forma cualitativa en la Figura 6 se muestran los contornos de temperatura en la mitad del segundo apile de ladrillos pasada 1 hora. Es evidente que no existe una variación apreciable en la distribución de esta variable entre los tres mallados considerados.

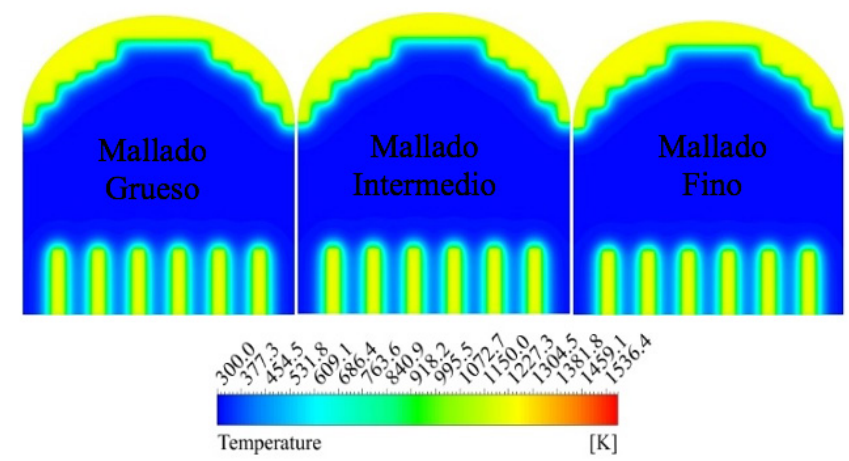

Figura 6. Contornos de temperatura al interior de los ladrillos para los 3 mallados.

Para todos los contornos de temperatura presentados en este trabajo, la escala de temperatura contemplada abarca de $300 \mathrm{~K}$ a $1575 \mathrm{~K}$, a menos de que se indique lo contrario.

En la Figura 7 se muestran las temperaturas y velocidades en función del tiempo usando los tres mallados contemplados en dos localizaciones. La primera de estas es entre los dos primeros apiles de ladrillos en el centro del tercer canal a una altura de $30 \mathrm{~cm}$, mientras que la segunda se localiza a la misma altura y posición transversal, pero en medio de los apiles 2 y 3 . Tal como se puede apreciar, las líneas de temperatura en ambos puntos para los 3 mallados se superponen casi en su totalidad, con diferencias máximas entre el mallado grueso y el fino de $0.16 \%$ y $0.28 \%$ para el punto $1 \mathrm{y}$ el punto 2 respectivamente. 
a)
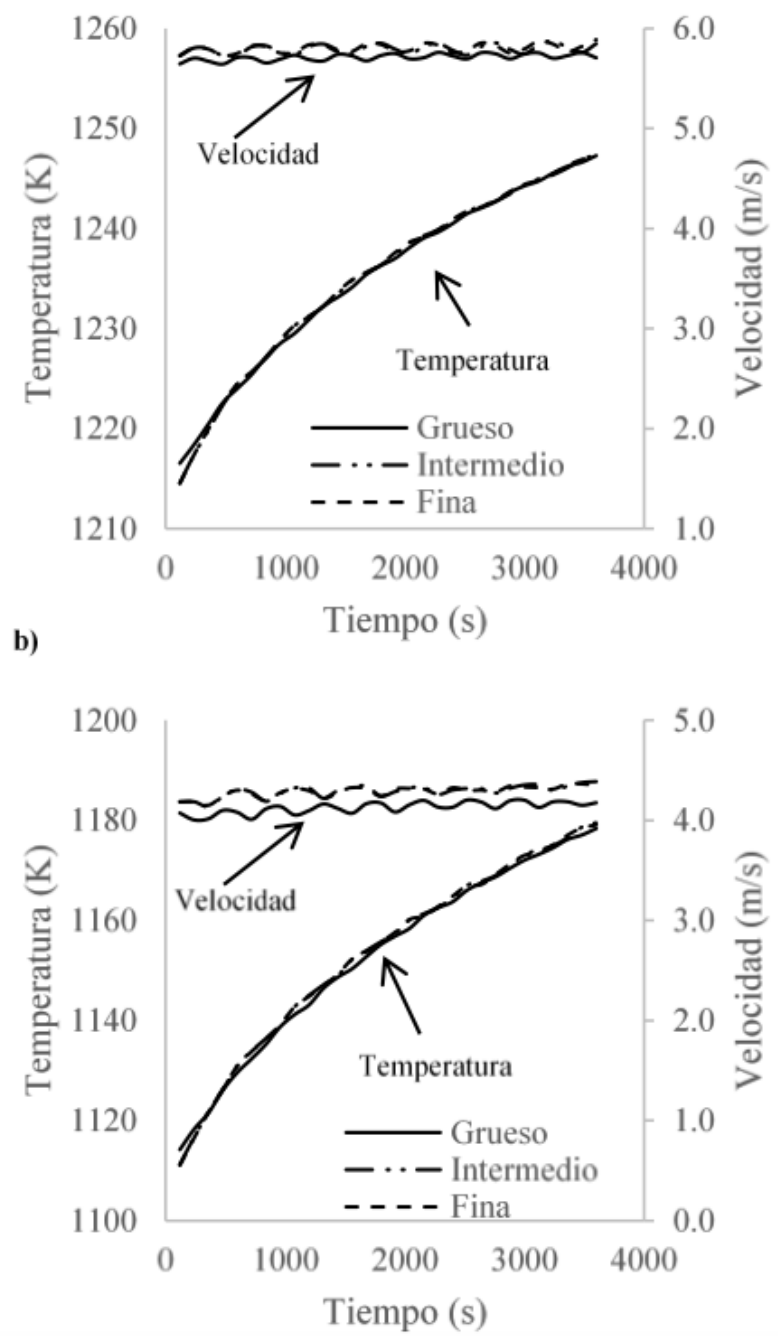

Figura 7. Comparación de temperatura y velocidad en puntos dentro del fluido. a) Punto 1. b) Punto 2.

Para todos los contornos de temperatura presentados en este trabajo, la escala de temperatura contemplada abarca de $300 \mathrm{~K}$ a $1575 \mathrm{~K}$, a menos de que se indique lo contrario.

En la Figura 7 se muestran las temperaturas y velocidades en función del tiempo usando los tres mallados contemplados en dos localizaciones. La primera de estas es entre los dos primeros apiles de ladrillos en el centro del tercer canal a una altura de $30 \mathrm{~cm}$, mientras que la segunda se localiza a la misma altura y posición transversal, pero en medio de los apiles 2 y 3 . Tal como se puede apreciar, las líneas de temperatura en ambos puntos para los 3 mallados se superponen casi en su totalidad, con diferencias máximas entre el mallado grueso y el fino de $0.16 \%$ y $0.28 \%$ para el punto 1 y el punto 2 respectivamente.

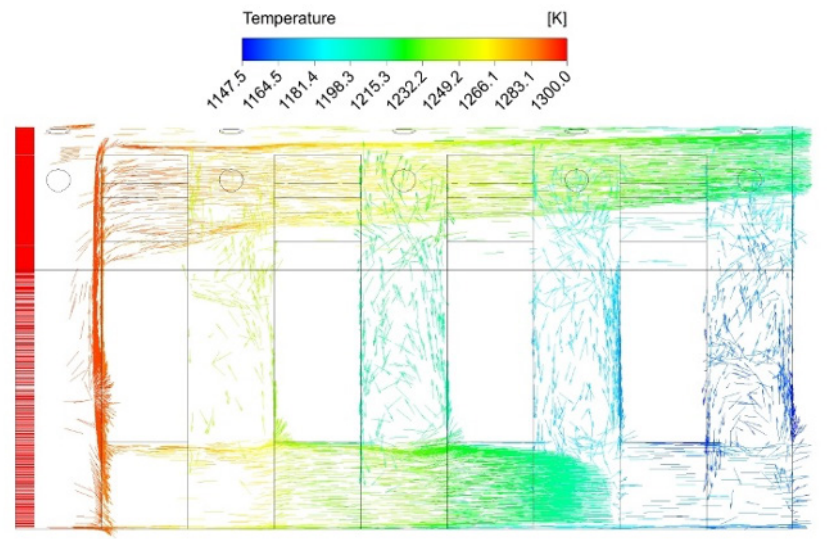

Figura 8. Líneas de corriente pasadas 5.2 horas.

Se puede apreciar como los gases que viajan por la parte inferior del horno se enfrían con mayor rapidez, producto de una menor cantidad y probablemente una velocidad mayor lo que aumenta la convección. Sin embargo, lo ideal sería que la tasa de reducción de temperatura de los gases fuese uniforme para conseguir un tratamiento similar en todos los ladrillos. Este comportamiento sugiere la necesidad de realizar un análisis de distribución de carga y configuración de los ladrillos que permita una mayor uniformidad. Entre los espacios de separación de los apiles se evidencia zonas de recirculación que pueden ayudar a uniformizar la temperatura sobre los ladrillos. No obstante, como se muestra posteriormente, al no tener canales para el flujo en esta zona, la recirculación no es suficiente para lograr un calentamiento uniforme de la carga.

En la Figura 9 se muestran los contornos de temperatura superficial de los ladrillos en diferentes instantes de tiempo. En cada caso los ladrillos del extremo derecho son los primeros en tener contacto con los productos de combustión. Es claro que a nivel superficial no existen gradientes de temperatura relevantes asociados con la ubicación de los ladrillos dentro de un mismo apile. Sin embargo, se observan diferencias de temperatura en el interior de los mismos, lo que puede afectar las propiedades del producto final.

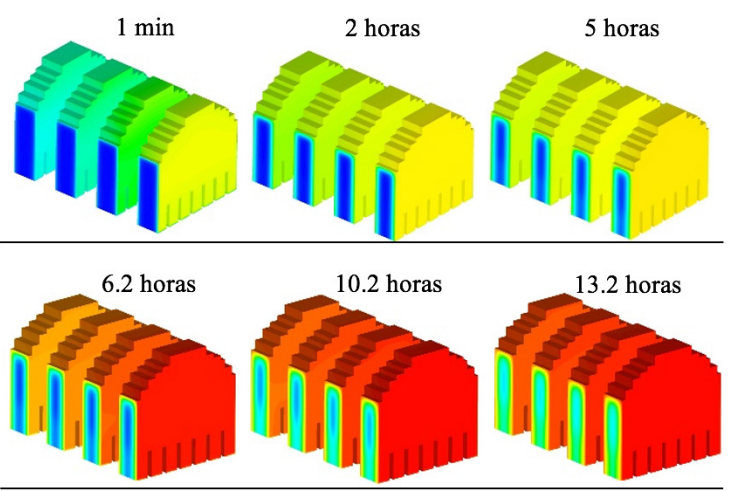

Figura 9. Contorno de temperaturas ladrillos. 
Las mencionadas variaciones internas pueden observarse con mayor detalle en la Figura 10, donde se muestra el contorno de temperaturas en plano transversal en la mitad del primer apile. Mientras la superficie de los ladrillos alcanza temperaturas entre 1100 y $1200 \mathrm{~K}$ pasadas más de 13 horas, el interior se encuentre entre 650 y $750 \mathrm{~K}$. Además, los ladrillos que fungen como pilares del apile formando los canales inferiores alcanzan temperaturas cercanas a los 1350 $\mathrm{K}$ en el interior, es decir incluso mayores a las temperaturas superficiales de la parte superior.

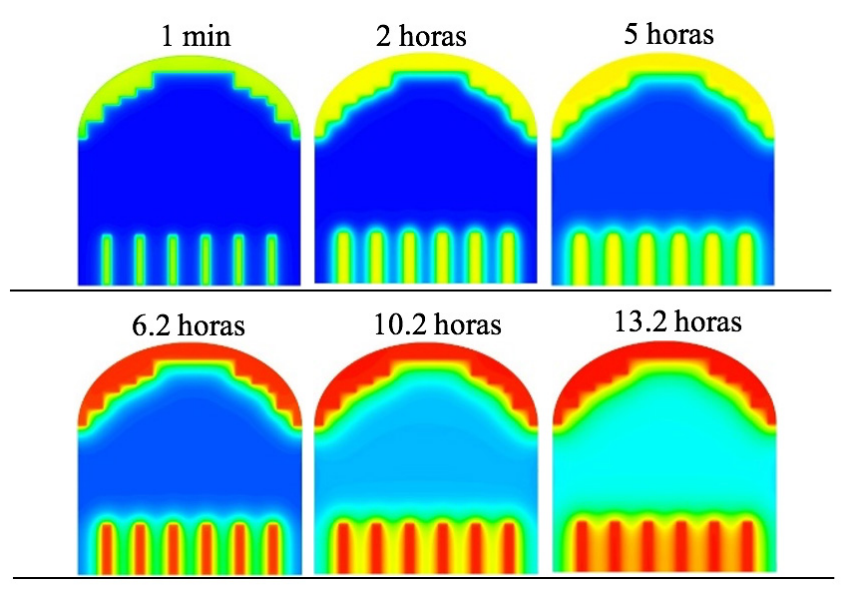

Figura 10. Temperaturas al interior de los ladrillos.

Para confirmar la no - homogeneidad al interior de los apiles en la Figura 11 se exponen los contornos de temperatura en un plano medio vertical en dirección longitudinal para los tiempos asociados con la etapa de cocción. Al cabo de las 13 horas las diferencias entre las temperaturas internas y superficiales son inferiores en comparación a la variación transversal, siendo en promedio la temperatura interna de $850 \mathrm{~K}$. Esta reducción en la diferencia de temperaturas puede asociarse un espesor menor y las zonas de circulación identificadas entre los apiles (ver Figura 8), aunque continúa siendo indeseable para la calidad y las propiedades finales de los ladrillos.

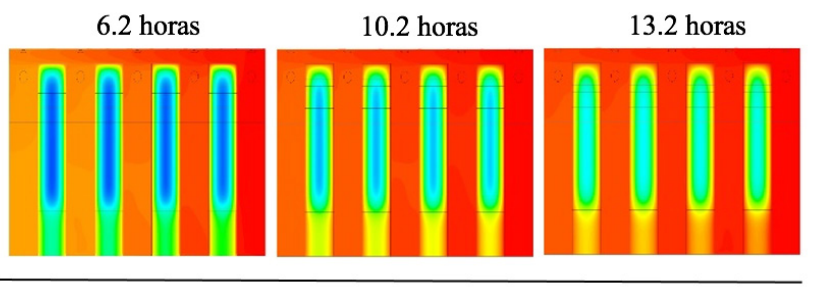

Figura 11. Temperaturas plano vertical longitudinal.

Tomando en conjunto los resultados fluidodinámicos y térmicos, se hace evidente que la distribución de los ladrillos al interior del horno puede no ser las más adecuada en términos del proceso de cocción, aunque su selección puede estar asociada con la cantidad de producto final requerida por la empresa. Si bien una propuesta de configuraciones alternativas requiere un estudio más detallado junto con los efectos sobre la productividad, es claro que la simulación permite identificar comportamientos difícilmente apreciables en un sistema real, teniendo en cuenta además que no es un horno de tipo laboratorio sino un equipo industrial.

\section{Conclusiones}

En el presente estudio se realizó la simulación de un horno Hoffman utilizado por la empresa Ladrillera Ocaña. Para ello se utilizó la mecánica de fluidos computacional (CFD) usando como dominio de cálculo una de las cámaras de cocción. A partir de esta fue posible obtener los perfiles térmicos y fluidodinámicos en la cámara, junto con la distribución de temperatura al interior de los ladrillos. Las líneas de corriente y la temperatura de los gases el interior del horno evidencia que el proceso de transferencia de calor ocurre de forma no uniforme, siendo aparentemente mayor en la zona inferior donde los gases se enfrían con mayor rapidez asociados a los canales más pequeños por los que fluye, produciendo un aumento en la velocidad que mejora la transferencia de calor por convección. La distribución de temperaturas de los ladrillos expone gradientes de temperatura importantes entre las temperaturas superficiales y el interior de estas, lo cual puede afectar las propiedades finales del producto. Finalmente, el comportamiento fluidodinámico encontrado sugiere que es necesario cambiar la distribución de los ladrillos para logar una mayor uniformidad en las temperaturas durante el proceso de cocción.

\section{Referencias}

[1] 2016 IEO, International Energy Outlook 2016. U.S. Department of Energy, 2016.

[2] L. Balza, R. Espinasa, and T. Serebrisky, Lights On?: Energy Needs in Latin America and the Caribbean to 2040. Inter-American Development Bank, 2016.

[3] Agencia Internacional de Energía, "Recomendaciones de Políticas de Eficiencia Energética Regionales," 2014.

[4] Research and Innovation in Advanced Combustion of Industrial Use - INCOMBUSTION, "Determinación del potencial de reducción del consumo energético en los subsectores manufactureros códigos CIIU 1018.” UPME, p. 209, 2014, [Online]. Available: http:// www.upme.gov.co/Estudios/2014/INFORME_III Caracterizacion_energetica_VerPub.pdf.

[5] A. Amell, L. Rubio, Y. Cadavid, and C. Echeverri, "Informe final del proyecto: Evaluación de las necesidades tecnológicas y planes de acción tecnológica para mitigación del cambio climático." Ministerio de Medio Ambiente y Desarrollo Sostenible, 2013.

[6] J.Campos, E. Lora, L.Meriño, I. Tovar, andA. Navarro, "Ahorro de energía en la industria cerámica," Un Proy. la unidad planeación Min. energética Colomb. y el Inst. Colomb. para el Desarro. la Cienc. y la Tecnol. 
Fr. Jose Caldas (Colciencias).(2014, octubre), 2010.

[7] T. S. Possamai, R. Oba, and V. P. Nicolau, "Numerical and experimental thermal analysis of an industrial kiln used for frit production," Appl. Therm. Eng., vol. 48, pp. 414425 , Dec. 2012, doi: 10.1016/j. applthermaleng.2012.05.025.

[8] C. A. G. Ubaque, J. C. M. Piraján, and M. L. V. Bohórquez, "Incineración de residuos sólidos municipales en hornos ladrilleros tipo Hoffman: simulación y análisis del flujo de gases," Tecnura, vol. 16, pp. 163-172, 2012.

[9] Y. Tu, H. Liu, S. Chen, Z. Liu, H. Zhao, and C. Zheng, "Effects of furnace chamber shape on the MILD combustion of natural gas," Appl. Therm. Eng., vol. 76(0), pp. 64-75, 2015, doi: 10.1016/j. applthermaleng.2014.11.007.

[10] Y. J. Liu, J. D. Li, R. D. K. Misra, Z. D. Wang, and G. D. Wang, "A numerical analysis of slab heating characteristics in a rolling type reheating furnace with pulse combustion," Appl. Therm. Eng., vol. 107, pp. 1304-1312, Aug. 2016, doi: 10.1016/j. applthermaleng.2016.07.074.

[11] G. Guerrero Gómez, "Hornos de combustión: alternativas de reducción de emisiones e incremento de rentabilidad," Bucaramanga: Universidad De Santander, 2017.

[12] H. Meng, G. Zhu, Y. Yu, Z. Wang, and J. Wu, "The effect of symmetrical perforated holes on the turbulent heat transfer in the static mixer with modified Kenics segments," Int. J. Heat Mass Transf., vol. 99, pp. 647-659, Aug. 2016, doi: 10.1016/J. IJHEATMASSTRANSFER.2016.03.110.

[13] S. A. Jácome Manzano, "Evaluación termodinámica del proceso de cocción y análisis de gases en hornos a cielo abierto y Hoffman en Ocaña (Universida)," 2012.

[14] T. Ishii, C. Zhang, and Y. Hino, "Numerical study of the performance of a regenerative furnace," Heat Transf. Eng., vol. 23(4), pp. 23-33, 2002, doi: 10.1080/01457630290090473.

[15] T. L. Bergman, F. P. Incropera, D. P. DeWitt, and A. S. Lavine, Fundamentals of heat and mass transfer. John Wiley \& Sons, 2011.

[16] A. Tehzeeb, M. Bhuiyan, and N. Jayasuriya, "Evaluation of brick Kiln performances using computational fluid dynamics (CFD)," Energy Environ. Eng., vol. 1(2), pp. 86-93, 2012.

[17] W. Ratanathavorn, S. Charoenjai, S. Janbuala, B. Chalermsinsuwan, and K. Poochinda, "Effects of Design Parameters for Clay Brick Kiln Using Computational Fluid Dynamics and Experimental Design.," Adv. Mater. Res., vol. 1101, 2015.

[18] A. Beyene, R. Venkata, and S. Getachew, "CFD Simulation of Biogas Fired Clay Brick Kiln,” Am. J.
Eng. Appl. Sci., vol. 11(2), pp. 1045-1061, 2017.

[19] S. Defaee Rad, A. Ashrafizadeh, and M. Nickaeen, "Numerical simulation of fluid flow and heat transfer in an industrial continuous furnace," Appl. Therm. Eng., vol. 117, pp. 263-274, May 2017, doi: 10.1016/J.APPLTHERMALENG.2017.02.031.

[20] O. Díaz-Ibarra, P. Abad, and A. Molina, "Design of a day tank glass furnace using a transient model and steady-state computation fluid dynamics," Appl. Therm. Eng., vol. 52(2), pp. 555-565, Apr. 2013, doi: 10.1016/J.APPLTHERMALENG.2012.11.018.

[21] C. Lezcano, J. Obando, and A. Amell, "Numerical simulation of a flameless combustion furnace with mixtures of methane and a sub-bituminous pulverized Coal," Combust. Sci. Technol., vol. 189(9), pp. 15911604, 2017, doi: 10.1080/00102202.2017.1305370.

[22] G. Guerrero-Gómez, E. Espinel-Blanco, \& T. Velásquez-Pérez, "Análisis isocinético y corrección a condiciones de referencia en horno a cielo abierto en el municipio de Ocaña, Norte de Santander", Revista Ingenio, vol. 14(1), pp. 43-51, jul. 2017. Doi: https:// doi.org/10.22463/2011642X.2194 